\title{
LOGÍSTICA HOSPITALAR: UMA SÍNTESE DO ESTADO DA ARTE
}

\author{
HOSPITAL LOGISTICS: A SYNTHESIS OF THE ART STATE
}

Renata Pereira Oliveira

Mestranda em Engenharia de Produção (Centro Federal de Educação Tecnológica Celso Suckow da Fonseca/ Brasil).E-mail: renatapoliveira@gmail.com.

\section{Augusto da Cunha Reis}

Doutor em Engenharia de Produção (Pontifícia Universidade Católica do Rio de Janeiro/Brasil). Professor (Centro Federal de Educação Tecnológica Celso Suckow da Fonseca/Rio de Janeiro).E-mail: augusto.reis@cefet-rj.br.

\section{Alexandre de Carvalho Castro}

Doutor em Psicologia Social (Universidade do Estado do Rio de Janeiro/Brasil). Professor (Centro Federal de Educação Tecnológica Celso Suckow da Fonseca/Rio de Janeiro).E-mail: o.aken@uol.com.br. 


\section{RESUMO}

Os sistemas de saúde visam a prevenção de doenças, promoção, assistência e recuperação da saúde da população. As preocupações gerais de saúde em um país são usualmente relevantes por se tratar de um direito do cidadão e pela necessidade de eficiência e qualidade na prestação do atendimento. Diante das dificuldades enfrentadas com as restrições de verbas e má administração, ocasionado principalmente por crise política econômica de um país, aumento da taxa de envelhecimento e complexidade do setor, torna-se importante diminuir os desperdícios e melhorar os processos nesta área. Este trabalho tem como objetivo realizar uma revisão sistemática da literatura sobre operações em logística hospitalar. Para tanto, pretende-se discutir a metodologia da revisão bibliográfica e analisar os resultados obtidos por meio de quatro bases de dados on-line, Web of Science, Science Direct, Emerald Insight e Scopus. Como resultado, os pesquisadores e gestores que trabalham no segmento da saúde terão uma síntese do estado da arte da logística hospitalar, com abordagens do uso da gestão lean, modelos computacionais, teoria das restrições, contratação de serviços de terceiros, armazenamento de materiais em redes, fluxo de pacientes, utilização de rôbos móveis, armários eletrônicos, RFID e tecnologia da informação, dentre outros.

Palavras-chave: Operações. Saúde. Logística.

\section{ABSTRACT}

Health systems aim at disease prevention, health promotion, care and recovery of the population. General health concerns in a country are usually relevant because it is a citizen's right and the need for efficiency and quality in care delivery. Faced with the difficulties faced by budget constraints and maladministration, caused mainly by a country's economic crisis, increasing the aging rate and complexity of the sector, it is becoming increasingly important to reduce waste and improve processes in this area. This paper aims to conduct a systematic review of the literature on hospital logistics in operations. To do so, we intend to discuss the methodology of the bibliographic review and analyze the results obtained through four online data base, Web of Science, Science Direct, Emerald Insight and Scopus. As a result, researchers and managers working in the health sector will have a art state synthesis of hospital logistics, with lean management approaches, computational models, constraint theory, contracting outsourced services, materials storage in networks, patients flow, use of mobile robotics, electronic cabinets, RFID and information technology, among others.

Keywords: Operations. Healthcare. Logistics. 


\section{INTRODUÇÃO}

O interesse da logística hospitalar para o campo da Engenharia, em especial, a Engenharia de Produção se dá em função da aplicação dos conhecimentos técnicos e gerenciais da área de Produção para otimizar o uso dos recursos humanos, materiais e tecnologia nos serviços logísticos para os sistemas de saúde. A síntese do estado da arte traz uma contribuição para que pesquisadores e estudiosos conheçam os conceitos e práticas abordados na literatura acadêmica, além do mapeamento dos trabalhos indexados de forma a responder aspectos e dimensões do campo de conhecimento pesquisado (FERREIRA, 2002).

Os sistemas de saúde são estruturas complexas e dispendiosas que enfrentam desafios frequentes e precisam se adequar à crescentes tecnologias e mudanças em seu ambiente (GOMES et al., 2016). Tais sistemas envolvem o atendimento de pacientes individuais, populações específicas e vários grupos de profissionais dentro do mesmo espaço. Há, portanto, uma necessidade de avaliar as abordagens tradicionais de gestão, os objetivos divergentes e experimentar novas estruturas para melhorar o nível do serviço entregue (AUBRY et al., 2014).

A área da saúde é justificada pela crescente demanda pelos serviços, aliado as limitações financeiras e a necessidade de prestação de serviços eficientes e de excelência (PATEL et al., 2015). Somado a isso, os recursos na área da saúde são escassos e geralmente sobrecarregados, o que implica em restrições ao sistema (ZHANG et al., 2015).

Segundo Wahab et al. (2016), a saúde é um dos principais motores para o desenvolvimento de um país e seu crescimento econômico, visto que dá suporte a melhoria da eficiência do trabalhador e, portanto, da sua produtividade. Acredita-se que o progresso nos indicadores de saúde poderia, no longo prazo, impulsionar o crescimento do PIB (Produto Interno Bruto) dos países.

Desde a virada do século XXI, a população Latino Americana tem aumentado e envelhecido. Entre os anos de 2000 e 2014, a população da região cresceu a uma taxa média de 19\%, passando de 526 para 626 milhões de habitantes. Neste mesmo período, o segmento da população acima de 65 anos aumentou de 29,3 para 46,3 milhões, o que equivale a um crescimento de $58 \%$, acima da média da população demonstrando um envelhecimento da mesma. Como em outras regiões do mundo, o efeito combinado de envelhecimento e crescimento da população coloca em risco a infraestrutura da saúde local e provoca desequilíbrios entre a capacidade e demanda, principalmente nas cirurgias eletivas e procedimentos de emergência em hospitais. Assim, o desafio para as próximas décadas é uma gestão de saúde eficaz e com qualidade (KETELHÖHN; SANZ, 2016). 
Nos países subdesenvolvidos ou em desenvolvimento, como o Brasil, a crise econômica aumenta as dificuldades enfrentadas pelos hospitais públicos com as restrições de verbas. Aliado a má gestão administrativa, falta aos pacientes atendimento digno e qualidade no nível de serviço prestado (PEREIRA, 2014).

A prestação de serviços hospitalares possui diferenças fundamentais com relação a outros tipos de atividades, principalmente no que diz respeito à atividade fim da organização - preservar e salvar a vida das pessoas. Considera-se que os materiais, recursos humanos, administração financeira e em especial a logística de suprimentos são os fatores críticos para o desenvolvimento de atividades de atenção à saúde e para a excelência operacional de uma organização hospitalar (RAIMUNDO et al., 2015).

A logística hospitalar é um dos desafios encontrados pelos gestores dos hospitais, principalmente no que diz respeito ao atendimento das necessidades organizacionais de forma rápida, correta e eficiente. O estudo e o planejamento dos processos logísticos que contemplam o abastecimento, compras, estoques e distribuição, podem auxiliar na redução e otimização dos recursos dos hospitais, desde materiais até pessoas, e assim impactar na redução dos custos. Esses processos são críticos e importantes na gestão de uma organização (SOUZA et al., 2013).

Com tudo isso, buscou-se fazer uma síntese na literatura acadêmica das discussões sobre aplicação da logística hospitalar com foco em operações, por meio de estudos, ferramentas e processos empregados. Para isso, pretendeu-se detalhar a metodologia para obter uma revisão teórica, informar a metodologia prática utilizada nesta pesquisa e analisar os resultados obtidos por meio de quatro bases de dados on-line, Web of Science, Science Direct, Emerald Insight e Scopus. Como resultado, os pesquisadores que atuam na área da saúde terão uma síntese das ferramentas e práticas mais utilizadas neste ambiente para melhorar os processos e aumentar a qualidade no atendimento.

O artigo está dividido em cinco seções, sendo esta a introdutória. A segunda seção apresenta a metodologia teórica e prática utilizada para desenvolvimento do trabalho. A terceira seção expõe a revisão sistemática da literatura com a síntese dos dados obtidos e análise de conteúdo sobre a aplicação da logística hospitalar. A quarta seção apresenta as considerações finais e a última seção exibe as referências utilizadas para compor este trabalho.

\section{METODOLOGIA}

A etapa metodológica de uma pesquisa é dada como importante em um trabalho, pois nela são definidos os meios pelos quais serão buscadas e obtidas as respostas aos problemas que ocorrem nas mais variadas 
áreas de conhecimento. Para que isto ocorra de maneira satisfatória é necessário que sejam selecionadas corretamente tanto a técnica quanto o método adotado (LUDWIG, 2012). Esta etapa também é responsável pelos métodos utilizados para coletar, processar e analisar os dados, de modo a garantir o rigor técnico e teórico para a obtenção de resultados aplicáveis e reais (STENDER, 2016).

O método de pesquisa aqui adotado consistiu em duas etapas: a primeira estabeleceu a revisão sistemática da literatura por meio dos critérios de seleção vinculados ao referencial teórico e a segunda etapa implicou a análise de conteúdo dos artigos tidos como mais representativos da área pesquisada.

Para a revisão sistemática da literatura, optou-se em recolher os dados exclusivamente a partir de bases online, uma vez que base de dados on-line são uma ferramenta importante na seleção de artigos de revistas científicas (ROWLEY et al., 2004), e acadêmicos e profissionais geralmente usam essas bases para coletar dados de pesquisa assim como divulgar novos resultados de investigação (REIS, 2013).

Foram escolhidas as bases de dados Science Direct, Emerald, Scopus e Web of Science, por disponibilizarem várias revistas científicas, efetivamente representativas do campo a ser pesquisado. Os critérios de inclusão definidos para a seleção foram artigos indexados nos referidos bancos de dados nos últimos doze anos (janeiro/2005 a fevereiro/2017). Para selecionar os artigos, uma pesquisa avançada foi realizada utilizando a expressão booleana "E" que combinou palavras-chave para melhores resultados específicos, tal como preconizado no Rowley et al. (2004). A definição das palavras-chave no processo de elaboração de um projeto de pesquisa é importante para cumprir com os requisitos necessários ao embasamento teórico e fortalecer os dados discutidos (STENDER, 2016).

O procedimento de investigação incluiu o termo hospital logistics no título do artigo e o termo operations em todos os campos, sem o uso das aspas, separados através da booleana "E", pois o interesse era analisar as práticas e ferramentas operacionais da logística hospitalar. O resultado da busca contemplou 44 (quarenta e quatro) artigos indexados.

Em um segundo momento, após a revisão sistemática da literatura, foi realizada a análise de conteúdo para classificar e apresentar os artigos. A análise de conteúdo permite aos investigadores selecionar, filtrar e resumir volumes de dados para sua análise. Esta técnica facilita a inferência objetiva e sistemática, o que permite a identificação das características relevantes de um determinado assunto, especialmente aqueles isolados por vários investigadores (GAO, 1996). Difere-se de outros métodos de revisão, pois busca superar possíveis vieses em cada uma das etapas, seguindo um método rigoroso de busca e seleção de pesquisas; avaliação de relevância e validade dos estudos encontrados; coleta, síntese e interpretação dos dados 
oriundos de pesquisa (SOUZA et al., 2010). Para tanto, realizou-se a leitura do título e resumo de todos os trabalhos encontrados, além da avaliação se os mesmos possuíam o conteúdo integral disponíveis nas bases e se estavam em inglês ou espanhol por serem línguas universais.

Nesta etapa foram descartados 08 (oito) artigos que não tinham o seu conteúdo total disponível e 13 (treze) que estavam em duplicidade nas bases. Posteriormente por meio da leitura na íntegra foram mantidos 24 (vinte e quatro) estudos que respondiam ao objetivo proposto e que serão apresentados na próxima seção.

Frente a esse quadro pode dizer que quanto ao método este artigo optou por uma pesquisa de abordagem qualitativa e de objetivo exploratório.

\section{REVISÃO SISTEMÁTICA DA LITERATURA}

Para efeito de clareza na exposição, optou-se por segmentar a síntese dos dados e a análise de conteúdo deles, conforme apresentado nos tópicos abaixo.

\subsection{SÍNTESE DOS DADOS}

Os resultados obtidos, após a busca nas bases de dados selecionadas, contemplaram 44 (quarenta e quatro) artigos indexados, conforme apresentado na Tabela 1. 
Tabela 1 - Resultado da busca de hospital logistics com foco em operations

\begin{tabular}{|c|c|c|c|c|c|c|c|c|c|c|c|c|c|c|c|}
\hline Ano (2000) & 05 & 06 & 07 & 08 & 09 & 10 & 11 & 12 & 13 & 14 & 15 & 16 & 17 & Total & (\%) \\
\hline Web of Science & & & 2 & & & 1 & & 2 & & 1 & & 2 & & 8 & 18,2 \\
\hline Emerald & & & 1 & & & & 2 & & & & & 1 & & 4 & 9,1 \\
\hline Science Direct & & & 1 & & & & 1 & & & 1 & 1 & 1 & 2 & 7 & 15,9 \\
\hline Scopus & & 1 & 2 & 2 & & & 2 & 2 & 4 & 3 & 2 & 6 & 1 & 25 & 56,8 \\
\hline Total & & & & & & & & & & & & & & 44 & 100 \\
\hline
\end{tabular}

Fonte: Elaboração dos autores

A Science Direct é uma plataforma de acesso de revistas científicas e e-books da Editora Elsevier. A busca avançada nesta base indicou 7 (sete) artigos. A Emerald é uma editora acadêmica de revistas e livros em diversas áreas. A busca avançada nesta plataforma, contemplou 4 (quatro) artigos. A Scopus é um banco de dados de resumos e citações de revistas que inclui diversas editoras. A busca avançada nesta base indicou 25 (vinte e cinco) artigos. A Web of Science por meio de assinatura junto à Thomson Reuters Scientific também oferece a busca em diversas editoras. Nesta base, foram obtidos 8 (oito) artigos como resultado.

A evolução temporal dos artigos indexados nas bases de dados é apresentada, conforme Gráfico 1, tendo a Scopus como destaque. 


\section{Gráfico 1 - Evolução temporal dos artigos indexados de hospital logistics com foco em operations}

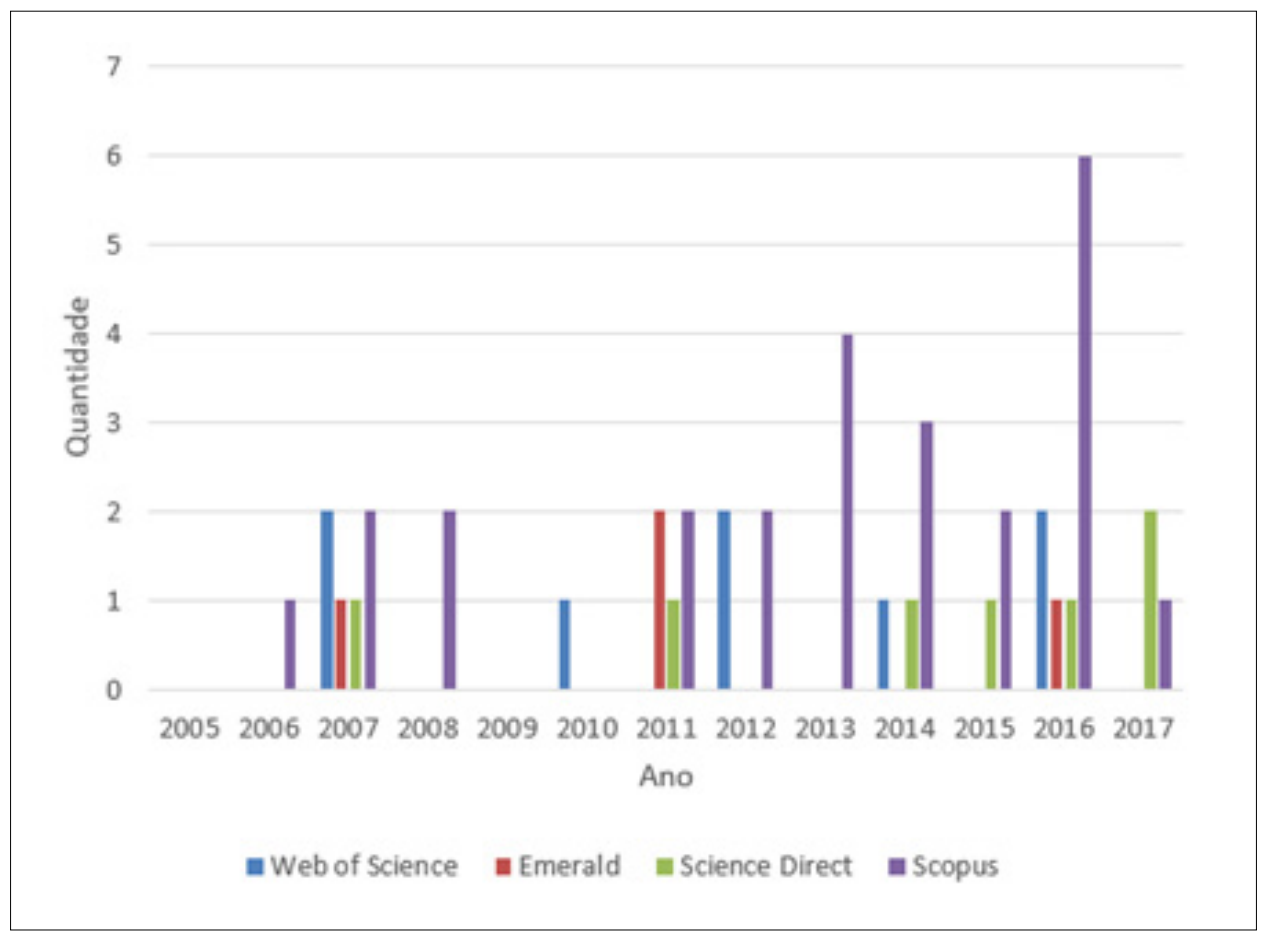

Fonte: Elaboração própria

Conforme análise da Tabela 1 e Gráfico 1, o ano de 2016 foi importante para publicações de trabalhos de logística hospitalar com foco operacional, tendo destaque as bases Scopus e Web of Science. A Emerald teve publicações isoladas. A Science Direct teve publicação em 2007, 2011 e a partir de 2014 contribuição anual sobre o assunto, sendo a base com maior representatividade em 2017. A Web of Science não teve linearidade em suas publicações, no entanto, foi a segunda maior base com artigos indexados sobre o assunto. Já a Scopus foi importante na produção acadêmica, o que se justifica pelo fato de ser uma base de dados que disponibiliza acesso a mais de 16.000 títulos de periódicos, mais de 1.200 revistas de livre acesso, mais de 500 anais de conferências, mais de 650 publicações comerciais e mais de 315 séries de livro (BIBENG, 2016). Esta última base teve destaque no ano de 2016 com a maior indexação de artigos, seguido do ano de 2013. Além disso, os anos de 2005 e 2009 não trouxeram nenhuma contribuição em todas as bases estudadas.

Os artigos selecionados após a revisão sistemática da literatura e análise de conteúdo são apresentados na Tabela 2. 
Tabela 2 - Artigos selecionados para análise, conforme objetivo proposto

\begin{tabular}{|c|c|c|c|c|}
\hline Ano & Número & Total & Referências & Fonte \\
\hline \multirow[t]{2}{*}{2017} & 1 & 2 & Volland et al. (2017a) & Omega \\
\hline & 2 & & Volland et al. (2017b) & European Journal of Operational Research \\
\hline \multirow[t]{8}{*}{2016} & 3 & 5 & Kriegel et al. (2016) & \\
\hline & & & & International Journal of Healthcare Management \\
\hline & 4 & & Ekeskär e Rudberg (2016) & Construction Management and Economics \\
\hline & 5 & & Salema e Buvik (2016) & \\
\hline & & & & International Journal of Procurement Management \\
\hline & 6 & & Aguilar-Escobar et al. (2016) & \\
\hline & & & & European Research on Management and Business Economics \\
\hline & 7 & & Law (2016) & Industrial Management \& Data Systems \\
\hline \multirow[t]{2}{*}{2015} & 8 & 2 & Bailey et al. (2015) & International Journal of Procurement Management \\
\hline & 9 & & Calderon et al. (2015) & Digital Communications and Networks \\
\hline \multirow[t]{2}{*}{2014} & 10 & 2 & Lee (2014) & IIE Transactions on Healthcare Systems Engineering \\
\hline & 11 & & Villa et al. (2014) & Health Policy \\
\hline \multirow[t]{3}{*}{2013} & 12 & 3 & Chiarini et al. (2013) & Leadership in Health Services \\
\hline & 13 & & Kriegel et al. (2013) & Logistics Research \\
\hline & 14 & & Shi et al. (2013) & Journal of Neurosurgery \\
\hline \multirow[t]{2}{*}{2012} & 15 & 2 & Brunero e Lamont (2012) & Scandinavian Journal of Caring Sciences \\
\hline & 16 & & Van Lent et al., 2012 & BMC Health Services Research \\
\hline 2011 & 17 & 3 & Liao e Chang (2011) & Expert Systems with Applications \\
\hline
\end{tabular}




\begin{tabular}{|c|c|c|c|c|}
\hline Ano & Número & Total & Referências & Fonte \\
\hline \multicolumn{3}{|c|}{18} & Bloss (2011) & Industrial Robot: An International Journal \\
\hline & \multicolumn{2}{|l|}{19} & Su et al. (2011) & $\begin{array}{l}\text { International Journal of Physical Distribution \& Logistics } \\
\text { Management }\end{array}$ \\
\hline 2010 & 20 & 1 & \multicolumn{2}{|r|}{ Surcical Endecrenve and Other Interventional Techniguec } \\
\hline 2008 & 21 & 1 & Van de Klundert et al. (2008) & Health Care Management Science \\
\hline \multirow[t]{3}{*}{2007} & 22 & 3 & Kuduvalli et al. (2007) & European Journal of Cardio-thoracic Surgery \\
\hline & 23 & & Lapierre e Ruiz (2007) & Computers and Operations Research \\
\hline & 24 & & Pan e Pokharel (2007) & Leadership in Health Services \\
\hline
\end{tabular}

Fonte: Elaboração própria

Os trabalhos apresentados na Tabela 2, que serão discutidos quanto ao seu conteúdo no tópico seguinte, foram realizados em várias regiões, sendo destaque no ambiente Europeu onde houve 10 (dez) estudos, especificamente na Áustria, Alemanha, Suécia, Inglaterra, Itália, Holanda, Espanha; seguido da Ásia com 6 (seis) publicações em Taiwan, Singapura e China; do continente americano com 4 (quatro) estudos nos Estados Unidos e Canadá; 1 (um) estudo na Austrália na Oceania e por fim, 1 (um) publicação na África, exclusivamente na Tanzânia. Apenas dois artigos não tiveram aplicação em uma determinada região, especificamente, os trabalhos de Volland et al (2017a) abordando a gestão da cadeia de abastecimento em hospitais e Volland et al (2017b) apresentando sugestões para diminuir o tempo dos enfermeiros com atividades logísticas.

As indexações ocorreram em diversas fontes como gerenciamento de construção, economia, negócios, sistemas de engenharia, logística, medicina, robótica, gestão healthcare, dentre outros, o que evidencia a multidisciplinariedade do tema.

A logística hospitalar pode ser dividida em dois grandes grupos: o primeiro atende o ambiente administrativo de um hospital, com o manuseio de material, integração com o fornecedor, dimensionamento de frota de ambulâncias, otimização de fluxo e cadeia de suprimentos, gestão de estoque e inovação logística e o 
segundo grupo atende a área assistencial com o objetivo principal de melhorar o fluxo de pacientes, através de métodos logísticos (AGUIAR; MENDES, 2016).

Os artigos que serão discutidos na próxima seção estão divididos nestes dois agrupamentos, onde 15 (quinze) artigos foram definidos pertencentes ao grupo administrativo e 9 (nove) ao assistencial, sendo apresentados nesta ordem. Não houve artigos classificados em ambos os grupos.

\subsection{ANÁLISE DE CONTEÚDO}

Conforme Pan e Pokharel (2007), mais de 30\% das despesas hospitalares totais são investidos em atividades logísticas e metade desse custo poderiam ser eliminados, através da melhoria de sua gestão. 0 desenvolvimento da cadeia de abastecimento em hospitais pode levar a melhor gestão farmacêutica e de inventário, relações com fornecedor aperfeiçoadas, pacientes mais satisfeitos e fluxo de trabalho eficaz para os funcionários do hospital. As duas principais abordagens para planejar atividades logísticas nos hospitais são: departamentos médicos solicitarem material sempre que o ponto de reabastecimento for atingido. Essa abordagem exige mais mão-de-obra, mais espaço no inventário e, obviamente, resultará em custo de operação. Já a abordagem orientada para a programação focaliza o desenvolvimento para lidar com as operações de compra. Nesta abordagem, os reabastecimentos, as atividades de compras e as entregas de fornecedores estão bem programadas, respeitando as disponibilidades e evitando os estoques. Observaram que, embora os hospitais prestem serviços essenciais e exijam níveis inesperados de estoque, as entregas just-in-time (JIT) podem ser usadas para minimizar seus custos, o que evidencia a necessidade e importância da colaboração dentro da cadeia de suprimentos.

Ainda segundo Pan e Pokharel (2007), os hospitais estudados têm pelo menos um almoxarifado central interno e boa parte relatam ter mais de uma área de armazenamento. Estes hospitais implementam sistemas ERP (Enterprise Resources Planning) para monitorar os níveis de estoque dos almoxarifados individuais e central, além do uso dos sistemas de informação para funções logísticas. Apesar de utilizarem terceirização para melhorar os níveis de serviços, seguem quatro métodos básicos de distribuição: entrega direta ao departamento para uso; entrega direta ao almoxarifado do departamento médico para uso posterior; entrega aos armazéns centrais e então ao departamento médico para o uso; e entrega direta ao almoxarifado central e entrega aos armazéns dos departamentos.

Precisamente sobre o armazenamento de material, Lapierre e Ruiz (2007) apresentam uma abordagem inovadora para melhorar a logística hospitalar, coordenando a aquisição e operações de distribuição. Sempre que houver a necessidade de reabastecimento para um produto nas unidades de cuidados médicos, um 
sinal é enviado ao centro de distribuição. A reposição é exigida e a quantidade enviada a unidade. Quando um determinado ponto limite para um produto for atendida, ordens de compra são gerados para aquisição por um fornecedor externo. O ciclo se completa quando os produtos encomendados para as fontes externas são recebidos no almoxarifado central. Para tornar mais eficiente o ponto de reabastecimento, tours são feitos nas unidades para rodadas de controle de distribuição. A partir disso, foi elaborado um algoritmo para definir a entrega dos produtos e a quantidade requerida. Foi calculado o tempo médio requerido por cada operação logística, como recebimento, manuseio, preparação e distribuição do reabastecimento, controle de inventário e da capacidade de armazenamento disponível no central e em cada unidade.

O trabalho de Van de Klundert et al. (2008) discute os problemas de otimização do fluxo de instrumentos esterilizáveis nos hospitais que podem ser resolvidos ao redesenhar processos para melhorar a disponibilidade de materiais e reduzir custos. A maior parte da redução de custos pode ser alcançada por medidas relativamente simples como a adoção de processos de trabalho uniformes, padronização de materiais, descontos de quantidade, dentre outros. No hospital estudado, os instrumentos esterelizáveis são colocados em estoque e agrupados em redes para serem utilizados em cirurgias gerais ou específicas e entregues no momento exato das cirurgias planejadas. Assim, há uma rede própria para cada tipo de operação, uma rede para todas as operações e uma híbrida, com uma rede específica e outra geral. Além disso, os instrumentos são mantidos no armazenamento dos serviços centrais de esterelização, havendo uma diminuição nos custos de transporte.

Para Bloss (2011), estudos mostraram que 30\% do tempo dos enfermeiros são gastos longe dos leitos e dos cuidados aos pacientes, pois atuam em atividades que não são da sua atividade-fim, como a busca por medicamentos, suprimentos, resultados de exames e outras tarefas logísticas. A fim de melhorar as atividades logísticas de um hospital nos Estados Unidos e minimizar o tempo gasto dos enfermeiros com este tipo de demanda, foi desenvolvido um robô móvel denominado TUG em busca de respostas para seus problemas logísticos. Assim, o robô atua na obtenção de roupas de cama limpas para os pacientes, prescrições de suprimentos para os postos de enfermagem, envio de bandejas de refeição, transporte de amostras de teste dos pacientes para os laboratórios, dentre outros. O robô pode indicar elevadores para o andar desejado, possuir abertura automática de portas, evitar os obstáculos no seu caminho e até mesmo anunciar sua chegada e pedir às pessoas para se afastar. Os TUGs possuem um mapa interno desenvolvido em CAD, além disso, tags de RFID podem ser colocados ao longo do caminho para facilitar sua navegação. Estes robôs eliminam a necessidade de pessoal à disposição para fazer entregas, estão disponíveis 24 horas por dia para as atividades logísticas e possibilitam uma diminuição significativa da despesa de pessoal, tendo um retorno sobre o investimento de $185 \%$. 
Outra abordagem do mesmo robô, agora em Singapura, foi através de Calderon et al. (2015) que indicam que a robótica está continuamente fazendo progressos no ambiente da saúde, seja em cirurgias, vigilância e também com o manuseio de materiais. O mercado de robô está em crescimento, pois vai de encontro ao projeto de casas, escritórios e hospitais do futuro. A segurança é um item importante ao se falar de robôs móveis, por isso o TUG proposto possui um mecanismo de bloqueio por impressão digital ou código de segurança autorizado. Além disso, o aumento da evolução tecnológica e equipamentos para melhorar o ambiente de trabalho dos profissionais de saúde podem ajudar no aumento da produtividade. O objetivo da aplicação deste robô é o mesmo citado anteriormente, isto é, melhorar o manuseio de materiais, alimentos, exames, dentro do hospital, permitindo a redução do custo anual e o tempo médio de retorno para o transporte hospitalar.

Análogo ao objetivo anterior, outra forma de reduzir o tempo dos enfermeiros com as atividades logísticas é proposto por Volland et al. (2017b), onde sugerem a introdução do assistente de logística para assumir as tarefas logísticas da equipe médica. Este profissional deve atuar em tarefas como reposições de estoque, recolha de roupa e de distribuição, preparação de alimentos, dentre outros, para permitir o aumento da eficiência dos enfermeiros no atendimento ao paciente, aumentando a satisfação do trabalho. Foi identificada a importância que os hospitais estimem o número de assistentes que serão necessários. Portanto, o trabalho em questão desenvolve uma formulação, através do programa MIP (Mixed-Integer Program) para integrar o agendamento de tarefas, respeitando as relações de precedências e para garantir que a oferta de recursos seja pelo menos equivalente a demanda.

O trabalho elaborado por Su et al. (2011) apresentou os resultados de reter a posse dos materiais de operações logísticas e encontrar formas inovadoras para melhorar o controle de inventário e reduzir o espaço de armazenamento, além do tempo de pessoal. A cadeia de fornecimento original compreendia as encomendas na segunda, quarta e sexta-feira e o recebimento da ordem, por meio de uma entrega interna nos mesmos dias. A encomenda era de acordo ao ponto de reabastecimento, isto é, sempre que o estoque era reduzido para um determinado ponto, o controlador de material encomendaria uma quantidade fixa. Para evitar problemas, um nível mais alto de estoque básico era estabelecido pelas unidades de saúde e departamento de logística. O projeto final prôpos um sistema de pedidos semanais onde todas as ordens de unidades de saúde fossem consolidadas na sexta-feira e transmitidas, através de um sistema informático. Os fornecedores deveriam entregar os produtos ao almoxarifado na quinta-feira para que os suprimentos que chegassem pudessem servir como amortecedor para evitar uma possível escassez. Utilizou-se do ciclo de feedback e brainstorming para chegar nestas conclusões. A tecnologia da informação também foi essencial para facilitar a integração e comunicação entre a cadeia de suprimentos e os parceiros. 
Além disso, cabe a logística hospitalar analisar o dimensionamento de frota de ambulâncias para o bemestar e a segurança dos pacientes, conforme abordado por Lee (2014). Uma ambulância enviada para uma chamada serve ao paciente no local e, se for necessário, o transfere para um departamento de emergência adequado e qualificado para o atendimento. Os quatro tipos de decisões de logística de ambulância estão associados com o tempo de resposta: localização da ambulância, realocação e despacho das mesmas, além da seleção hospitalar que deve ser feita de forma a evitar aglomerações e confirmar a disponibilidade de leito disponível. O objetivo da pesquisa foi desenvolver uma nova política de seleção hospitalar que pode levar a reduções significativas do tempo de resposta, isto é, intervalo entre chegada ao hospital e a hora em que a ambulância se torna disponível para responder a outra chamada. A proposta foi integrar três princípios de decisão: proximidade, congestionamento e centralidade. A política também enfatizou o papel da tecnologia da informação na medição e comunicação das informações. Dessa forma, parâmetros de ajuste com indicação do peso adequado aos princípios de decisão foram utilizados.

A revisão da literatura também indicou as interações logísticas em projetos de construção em hospitais, através de Ekeskär e Rudberg (2016). O estudo teve o objetivo de explorar o uso de fornecedores de terceiros para projetos de construção em um hospital da Suécia e analisar os seus efeitos. O hospital em questão possui grandes obras em curso com novos edifícios sendo construídos, bem como existentes sendo remodelados. Portanto, há exigências de que o processo de construção não deva perturbar as operações ao redor do hospital. O uso da gestão da cadeia de abastecimento (SCM) foi um meio para lidar com sua característica complexa e de natureza temporária. A contratação de fornecedores de terceiros facilitou um aumento no trabalho produtivo no próprio canteiro de obras, redução dos custos e uma maior utilização dos ativos do local. Por outro lado, o estudo também mostrou uma falta de SCM entre os atores envolvidos no projeto, impedindo-os de colher todo o potencial deste tipo de contratação. A competência logística externa do prestador também permitiu que o projeto se concentrasse na construção ao manusear a cadeia de abastecimento por meio de regulamentações para otimizar a logística.

Ainda na visão de contratação de serviço, Kriegel et al. (2013) estudam como fornecer cuidados médicos orientados para as necessidades do paciente. É preciso que o produto certo esteja no lugar certo, no momento adequado para o cliente definido, na quantidade e qualidade requerida e ao menor custo possível. A fim de obter uma visão geral da situação atual do mercado e para ver as oportunidades e riscos para os serviços de logística, foi realizado um estudo com diferentes prestadores de serviços logísticos em variados departamentos, como farmacêutico, esterilização, alimentar, material, etc. Os resultados do estudo mostram que, por um lado, os atuais serviços da cadeia de abastecimento aos hospitais envolvem principalmente campos logísticos hospitalares de médio-baixo prioridade do ponto de vista de decisores do hospital. 
Por outro lado, mostram que prestadores de serviços externos concentram-se mais oferecendo serviços abrangentes de contrato. Além disso, também foi identificado uma tendência para contratação de serviço externo para atender a demanda logística.

O estudo de Salema e Buvik (2016) examinam a associação entre a integração do comprador-fornecedor e o desempenho logístico deste relacionamento. Embora a maioria dos profissionais associe problemas de disponibilidade de medicamentos à falta de recursos financeiros, um recente estudo mostra que a falta ocorre pela indisponibilidade dos itens e falhas no sistema. A implicação é que uma empresa deva estar mais consciente da necessidade de integração da cadeia de abastecimento, sincronizando as competências logísticas essenciais, além do intercâmbio de coaching e compartilhamento de conhecimento estratégico. O trabalho prôpos uma integração funcional cruzada, onde as atividades logísticas interagem com outras áreas funcionais, levando ao desencorajamento do comportamento individualista e ao encorajamento do comportamento que reduz a otimização e os conflitos. A melhoria da integração funcional cruzada simplifica as práticas operacionais, tais como procedimentos e métodos padronizados e reduz a incerteza e complexidade associadas às operações gerenciais.

Já Liao e Chang (2011) estabaleceram um modelo de simulação para a cadeia de suprimento do sistema logístico hospitalar baseado no método dinâmico de Taguchi. O método de Taguchi usa relação sinalruído (SN) para avaliar robustez e variação de um sistema. O trabalho propõe uma abordagem ótima, incluindo a rede neural e o algoritmo genético para obter um design robusto na obtenção de desempenho do departamento farmacêutico. Foi verificado que a capacidade de contratação hospitalar e farmacêutica torna-se mais difícil quando a demanda flutua ou quando a capacidade do produto é limitada. Além disso, a característica just-in-time pode estabelecer uma boa relação entre o fornecedor e contratante.

O estudo de Law (2016) explorou a situação atual da logística de medicamentos e dos centros de operações de medicamentos em hospitais públicos na China. As organizações estão constantemente enfrentando desafios em relação às mudanças na produção ou logística de serviços. Com a concorrência feroz no mercado de saúde, serviço de qualidade, eficiência operacional e controle de custos tornaram-se tópicos essenciais nos hospitais. A otimização da gestão da logística de medicamentos não só leva ao menor custo operacional, mas também melhora a eficiência do hospital / clínicas e a qualidade do serviço. A fim de manter o bom desempenho, parceria entre fornecedores e clientes são cruciais. Além disso, o desempenho da operação interna também deve ser considerado, como sistema de suprimentos, fluxo de materiais e fatores humanos.

Para Bailey et al. (2015), o tema principal foi a colaboração de hospital-fornecedor alcançar cadeias de abastecimento otimizadas que promovam a transparência e a comunicação como meios para superar 
os custos crescentes e satisfazer as expectativas de qualidade. Esses conceitos visam dar visibilidade do inventário para fornecedores, reduzindo a incerteza, os prazos e a necessidade de segurança, o que resulta em práticas de cadeia de suprimento mais rentáveis, como o just-in-time. Outro tema abordado foi o o processo de reengenharia com a utilização de novas tecnologias de informação e comunicação (TIC), tais como codificação de barras e identificação por radiofrequência. O objetivo foi que fornecedores fizessem entregas diretas para unidades de cuidados com o paciente. Para tanto, apresentou-se o conceito de um banco de armários eletrônicos. Cada banco de armários compreende numerosas caixas seguras, equipadas com comunicações sem fio (3G) para notificação das entregas confirmadas aos destinatários. Eles são tipicamente operados e mantidos pelo fornecedor e situados em locais centrais. Essas caixas são apenas um meio de detenção temporária de ações (máximo de um dia), informando ao pessoal que uma encomenda específica está pronta a recolher. A caixa de armários envia uma mensagem para a placa de switch do hospital que a transmite, juntamente com os códigos de segurança, através do sistema de telefonia, conforme Figura

1. Essa solução permite aumento da velocidade e qualidade dos cuidados com a saúde.

\section{Figura 1 - Uso da tecnologia da informação para entrega de materiais}

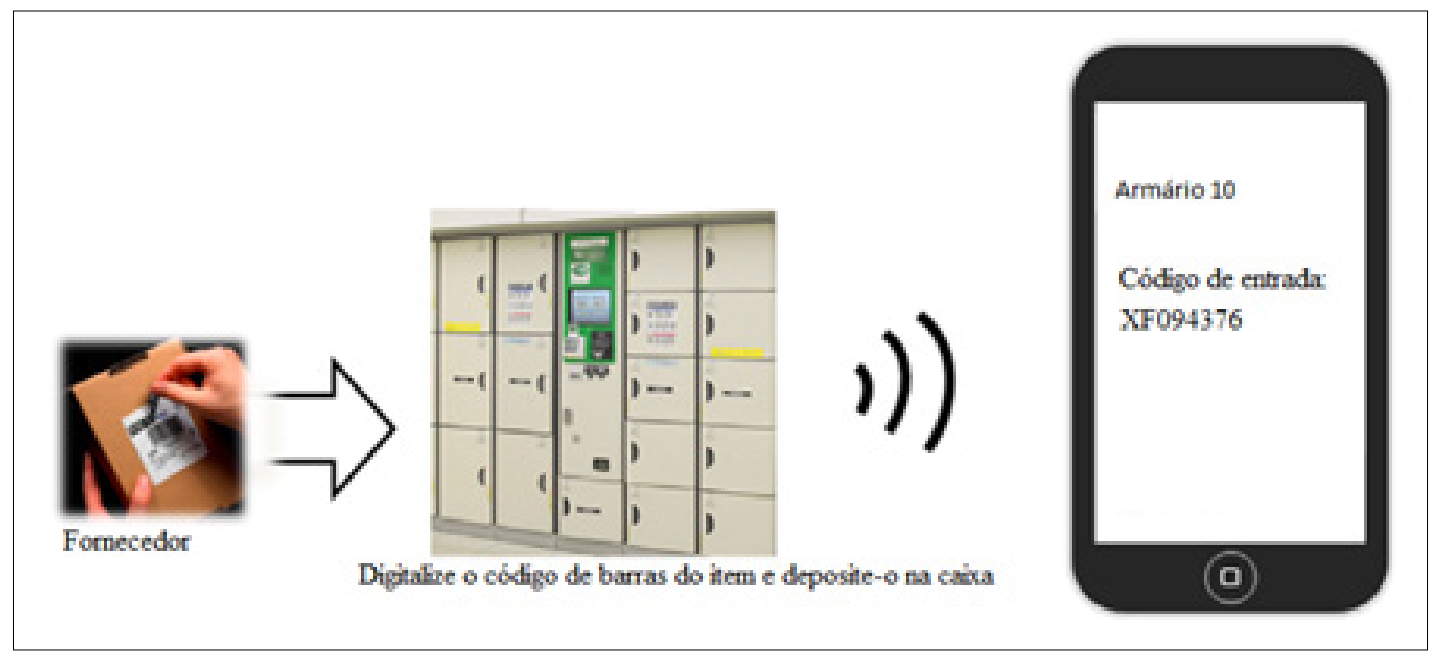

Fonte: Adaptado de Bailey et al. (2015)

Por último, o estudo de Volland et al. (2017a) realizou uma revisão da literatura sobre a gestão da cadeia de abastecimento em hospitais. Foi verificada a necessidade de minimizar os custos de aquisição, através 
de alianças de compras para aumentar a quantidade, dar maior transparência aos preços e criar limites máximos, por meio de contratos pré-fixados. Para materiais de alto valor, o uso de RFID foi indicado para melhor rastreabilidade. Além disso, verificou-se a necessidade de ter um sistema indireto com um armazém central que receba a demanda de fornecedores e envia os itens para o ponto de uso para posterior assistência ao paciente; o sistema semi-direto, onde os fornecedores entregam diretamente no ponto de uso e o direto, que está mais próxima do just-in-time, onde a entrega é direta para o cuidado do paciente. Segundo a revisão, observou-se ser comum manter estoque de segurança "escondido" nas unidades de saúde, devido as dificuldades na implementação de estruturas organizadas. Ademais, também verificou-se que terceirizar a distribuição de itens não-críticos é uma escolha viável, permitindo que o pessoal se concentre nas atividades de atendimento ao paciente.

Os conceitos de logística têm sido implementados em diversas indústrias, mas sua transferência para o ambiente healthcare ainda não tem sido intensificado na literatura. Muitas publicações concluem que geralmente os conceitos são aplicados a saúde, mas há obstáculos que precisam ser vencidos, como a aplicabilidade de lean thinking, six sigma, kanban, $5 \mathrm{~S}$ e teoria das restrições. Foi identiticado cinco áreas para pesquisas futuras baseado na revisão da literatura: o uso da tecnologia da informação; influência de diferentes partes interessadas e entre prestadores de serviços de saúde; pontos fortes e fracos da filosofia de gestão lean; gerenciamento de processos de negócios; métricas de desempenho e técnicas de cadeia de abastecimento não apenas à logística, mas também ao fluxo do paciente.

O fluxo de paciente supracitado, está diretamente relacionado aos artigos classificados no grupo assistencial, conforme análise a seguir.

A logística de pacientes oferece a possibilidade de melhorar a qualidade no atendimento e o uso dos recursos no hospital. Para tanto, a melhoria dos canais de comunicação assume importância, a fim de possibilitar o intercâmbio de informações, interfaces do ambiente e transporte do paciente. Percebe-se que nos hospitais estudados não há uma orientação para logística dos pacientes de forma clara e bem definida. A fim de melhorar este processo é proposto o uso de métricas de indicadores qualitativos e quantitativos para obter o tempo de espera dos pacientes, desempenho conceitual, gestão da qualidade. Assim, com base em horizonte de tempo (mês/ano) é possível representar o volume de pacientes, visualizar entradas e saídas, bem como o respectivo custo para unidades de produção. Além disso, é proposto o diagrama de ishikawa para servir como base para discussão do desenvolvimento de soluções adequadas (KRIEGEL et al., 2016).

O tempo de internação hospitalar, após uma determinada cirurgia, também deve ser considerado na logística de pacientes, segundo Kurian et al. (2010). Já Van Lent et al. (2012) indica que abordagens promissoras 
como benchmarking, pesquisa operacional, gestão lean e six sigma, poderiam ser adotados para melhorar a logística do paciente em saúde. Segundo a pesquisa, os fluxogramas foram os mais comumente utilizados, em 94\% dos hospitais. Além disso, a maioria dos hospitais apoiam os processos e educação da logística de pacientes, porém apenas 24\% tinham treinamento interno permanente para este fim.

Outro método utilizado para a logística de pacientes foi a regressão logística que tem como objetivo produzir, a partir de um conjunto de observações, um modelo que permita a predição de valores tomados por uma variável categórica. Neste caso, os estudiosos desejavam analisar a previsão de mortalidade intra-hospitalar, após cirurgia de válvula aórtica, a segunda operação cardíaca mais comum no Reino Unido. Portando, foi desenvolvido um modelo multivariável de previsão para ajudar no cálculo de risco específico de pacientes e ajustar o número de mortalidade para fins comparativos (KUDUVALLI et al., 2007).

Ainda sobre a regressão logística, Shi et al. (2013) comparam modelos de Redes Neurais Artificiais (RNAs) para predizer a mortalidade intra-hospitalar, após cirurgias no cérebro e comparar o modelo com a regressão logística. Os RNAs são sistemas computacionais inspiridos pelo sistema nervoso central que computam valores de entrada e são capazes de reconhecer padrões. Os dados dos estudiosos mostraram que o modelo RNA apresentou melhor desempenho se comparado a regressão logística. Por outro lado, indicaram que a precisão, sensibilidade e especificidade do modelo em questão são comparáveis ao padrão da regressão.

Já Villa et al. (2014) abordaram o gerenciamento do fluxo de pacientes que pode ocorrer em diversas unidades de produção hospitalar, como o departamento de emergência, salas de cirurgias ou clínicas ambulatoriais. Através do estudo realizado, verificaram que falha neste gerenciamento pode ser origem para vários problemas, a exemplo da variabilidade da carga de trabalho, erros e colocação de pacientes em situações inapropriadas. Os problemas de fluxo do paciente ocorrem quando as fontes, como camas, salas cirúrgicas e pessoal de enfermagem são alocadas de forma fixa e não são atualizadas regularmente para refletir a realidade. Além disso, foi apresentado como problema: escassez de capacidade em desequilíbrio com a demanda; variabilidade do fluxo do paciente levando a picos e depressões e falta de coordenação resultando em gargalos ou sub-utilização. Para minimizar estes problemas, os gerentes precisaram liderar estratégias com uma visão de $360^{\circ}$, através de um escritório centralizado para lidar com o fluxo de pacientes, além de estabelecer controle deste fluxo em tempo real, apresentando toda a jornada de cada paciente no hospital, desde o primeiro acesso até a sua alta.

Outro problema identificado por Aguilar-Escobar et al. (2016) foi a gestão dos prontuários em um hospital. Dado que este processo é circular, a prevenção de gargalos se torna especialmente importante. Assim, o 
objetivo da pesquisa foi analisar a aplicação da Teoria de Restrições (TOC) na logística de registros médicos em hospitais. A Teoria das Restrições é um paradigma de gestão com base na eliminação das restrições do sistema (ou estrangulamentos) que impedem que o fluxo produtivo seja capaz de satisfazer as exigências. O TOC é implementado com sucesso em organizações, principalmente em empresas e indústrias. Embora escasso, é no setor da saúde que as aplicações têm sido mais desenvolvidas, principalmente para a gestão do fluxo de pacientes. No caso de um hospital público, o interesse dessa aplicação é maximizar os serviços médicos de alta qualidade prestados aos clientes e, no futuro, sujeito a duas condições, o cumprimento das restrições orçamentárias e proporcionar ambientes de trabalho seguros e satisfatórios para os funcionários.

Uma outra abordagem apresentada por Brunero e Lamont (2012) referiu-se a supervisão clínica como um processo para melhorar as práticas da enfermagem. Para tanto, avaliou a implementação da supervisão em diferentes especialidades em um hospital da Austrália. A supervisão clínica é um processo de apoio profissional e aprendizado em que os enfermeiros são assistidos em sua prática, através de profissionais especializados ou por colegas. Verificou-se que apesar de haver uma falta de apoio de enfermeiros seniors, esta prática pode aumentar a satisfação do trabalho pelos funcionários e aumentar a qualidade dos serviços prestados.

Por fim, Chiarini (2013) aborda o transporte de pacientes dentro de grandes hospitais públicos e seus custos. Estes custos são muitas vezes relacionados ao transporte dentro de departamentos, enfermarias e clínicas ambulatoriais. O objetivo do trabalho foi demonstrar, através de um estudo de caso qualitativo, como ferramentas do lean thinking, como gráfico de espaguete, mapeamento de fluxos de valor e planilha de atividades, podem ajudar a reduzir os custos relacionados ao transporte de pacientes e outros tipos de resíduos. Foi identificado que quanto maior a rota percorrida por um paciente dentro do hospital, maior o custo do transporte, a possibilidade de contrair doenças e a perda de conforto, proporcionando um aumento da insatisfação dos pacientes. Para aplicar as ferramentas lean sugeridas, foi analisada a entrada de um paciente em traumas em um hospital da Espanha. Através de um diagrama de espaguete, foi verificado que um paciente que chega na emergência realiza uma triagem e em seguida o mesmo percorre $80 \mathrm{~m}$ para o primeiro exame ortopédico (FOE). Após isso, o paciente move 460m para a radiologia e depois em direção ao especialista ortopédico que deve hospitalizar o paciente ou dar alta. A Figura 2 indica este fluxo. 
Figura 2 - Fluxo de paciente de um hospital da Espanha - Diagrama de espaguete

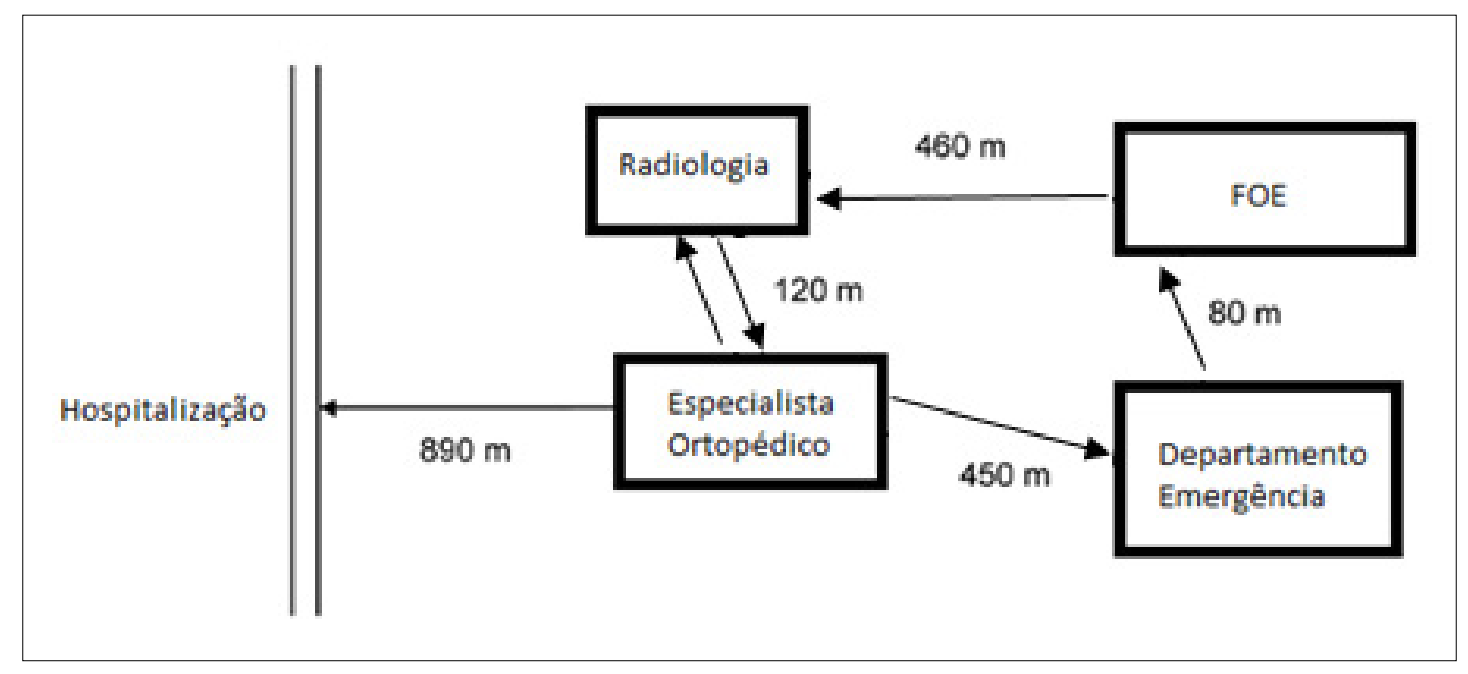

Fonte: Adaptado de Chiarini (2013)

Com o auxílio do mapa do fluxo de valor foi possível analisar e medir todos os tempos esperados, em função das filas e transporte dos pacientes dentro do hospital. A fim de reduzir distâncias e tempos, indicou-se as seguintes soluções: mover o departamento de FOE para o departamento de emergência. Deste modo, o caminho do paciente é reduzido em $80 \mathrm{~m}$ e por cinco minutos de transporte. Além disso, a triagem do trauma é feita por médico que ao mesmo tempo pode fazer o FOE, o que reduz o tempo médio de atendimento. 0 médico que tem a tarefa de examinar o paciente após a radiografia foi movido para dentro do departamento de radiologia. Essas melhorias reduziram o tempo de transporte em 30 minutos e eliminaram o tempo de espera de 20 minutos. Os resultados obtidos reduziram o tempo médio de espera do paciente do departamento de emergência para internação ou alta, reduziram custos e aumentaram a satisfação dos usuários e profissionais (CHIARINI, 2013).

Contudo, pode-se resumir na Tabela 3 as ferramentas, técnicas e práticas de logística hospitalar mais usados na síntese do estado da arte analisada. 
Tabela 3 - Resumo das ferramentas, técnicas e práticas de logística hospitalar

\begin{tabular}{|c|c|c|c|}
\hline Item & Ferramentas/Técnicas/ Práticas & Benefícios & Referência \\
\hline \multirow{3}{*}{1} & \multirow{3}{*}{ Just-in-time } & \multirow{3}{*}{ Minimizar custos e reduzir desperdícios. } & Pan e Pokharel (2007) \\
\hline & & & Liao e Chang (2011) \\
\hline & & & Bailey et al. (2015) \\
\hline 2 & Sistemas ERP & Monitorar os níveis de estoque. & Pan e Pokharel (2007) \\
\hline 3 & Tours & Controlar a distribuição dos materiais. & Lapierre e Ruiz (2007) \\
\hline \multirow[t]{2}{*}{4} & \multirow{2}{*}{$\begin{array}{l}\text { Coordenar o ponto de } \\
\text { reabastecimento }\end{array}$} & \multirow{2}{*}{$\begin{array}{l}\text { Permitir atividades de compras e entregas de } \\
\text { fornecedores bem programadas, respeitando as } \\
\text { disponibilidades e evitando os estoques. }\end{array}$} & Pan e Pokharel (2007) \\
\hline & & & Lapierre e Ruiz (2007) \\
\hline 5 & Processo de trabalho uniforme & $\begin{array}{l}\text { Reduzir custos, através da padronização de } \\
\text { processos. }\end{array}$ & Van de Klundert et al. (2008) \\
\hline 6 & Materiais agrupados em redes & Otimizar o fluxo de distribuição dos materiais. & Van de Klundert et al. (2008) \\
\hline \multirow[b]{2}{*}{7} & \multirow[b]{2}{*}{ Utilização de robôs móveis } & \multirow{3}{*}{$\begin{array}{l}\text { Diminuir o tempo gasto dos enfermeiros com } \\
\text { processos logísticos. }\end{array}$} & Bloss (2011) \\
\hline & & & Calderon et al. (2015) \\
\hline 8 & Inclusão de um assistente logístico & & Volland et al. (2017b) \\
\hline 9 & Utilização do programa MIP & $\begin{array}{l}\text { Integrar o agendamento de tarefas e garantir } \\
\text { oferta de recursos equivalente a demanda. }\end{array}$ & Volland et al. (2017b) \\
\hline \multirow[t]{3}{*}{10} & \multirow[t]{3}{*}{ Sistema de pedidos semanais } & $\begin{array}{l}\text { Melhorar o controle do almoxarifado e reduzir o } \\
\text { espaço de armazenamento. }\end{array}$ & Su et al. (2011) \\
\hline & & $\begin{array}{l}\text { Facilitar a integração e comunicação entre a } \\
\text { cadeia de suprimentos e os parceiros. }\end{array}$ & Su et al. (2011) \\
\hline & & $\begin{array}{l}\text { Facilitar a comunicação entre ambulâncias e } \\
\text { hospitais que possuem leitos disponíveis. }\end{array}$ & Lee (2014) \\
\hline \multirow[t]{2}{*}{11} & \multirow[t]{2}{*}{ Uso da tecnologia da informação } & $\begin{array}{l}\text { Possibilitar o armazenamento temporário de } \\
\text { material em armários eletrônicos, além da } \\
\text { comunicação para notificar as entregas. }\end{array}$ & Bailey et al. (2015) \\
\hline & & $\begin{array}{l}\text { Melhorar a rastreabilidade de material de alto } \\
\text { valor com o uso do RFID. }\end{array}$ & Volland et al. (2017a) \\
\hline 12 & Feedback & Melhorar a comunicação. & Su et al. (2011) \\
\hline 13 & Brainstorming & $\begin{array}{l}\text { Compartilhar ideias e estimular a participação } \\
\text { da equipe. }\end{array}$ & Su et al. (2011) \\
\hline
\end{tabular}


(conclusão)

\begin{tabular}{|c|c|c|c|}
\hline Item & Ferramentas/Técnicas/ Práticas & Benefícios & Referência \\
\hline 14 & $\begin{array}{l}\text { Contratação de serviço externo } \\
\text { para a demanda logística }\end{array}$ & $\begin{array}{l}\text { Facilitar a gestão dos serviços por especialistas, } \\
\text { reduzir custos operacionais e dividir } \\
\text { responsabilidades. }\end{array}$ & $\begin{array}{l}\text { Kriegel et al. (2013) } \\
\text { Volland et al. (2017a) }\end{array}$ \\
\hline 15 & $\begin{array}{l}\text { Interação da cadeia de } \\
\text { abastecimento com outras áreas } \\
\text { funcionais }\end{array}$ & $\begin{array}{l}\text { Simplificar as práticas operacionais, reduzir } \\
\text { conflitos e compartilhar conhecimento } \\
\text { estratégico. }\end{array}$ & Salema e Buvik (2016) \\
\hline 16 & $\begin{array}{l}\text { Melhoria dos canais de } \\
\text { comunicação }\end{array}$ & $\begin{array}{l}\text { Permitir o intercâmbio de informações, melhorar } \\
\text { a qualidade no atendimento e uso dos recursos } \\
\text { do hospital. }\end{array}$ & Kriegel et al. (2016) \\
\hline 17 & Diagrama de ishikawa & $\begin{array}{l}\text { Ajudar na identificação da causa raiz de um } \\
\text { problema. }\end{array}$ & Kriegel et al. (2016) \\
\hline 18 & Benchmarking & Obter melhores práticas. & Van Lent et al. (2012) \\
\hline 19 & Gestão lean & $\begin{array}{l}\text { Reduzir desperdícios e custos, melhorar a } \\
\text { qualidade das operações e proporcionar maior } \\
\text { eficiência nos processos. }\end{array}$ & $\begin{array}{l}\text { Van Lent et al. (2012) } \\
\text { Chiarini (2013) }\end{array}$ \\
\hline 20 & Treinamento & Desenvolver as habilidades da equipe. & Van Lent et al. (2012) \\
\hline 21 & Regressão logística & $\begin{array}{l}\text { Permitir a predição de valores estatísticos para } \\
\text { comparar dados. }\end{array}$ & $\begin{array}{l}\text { Kuduvalli et al. (2007) } \\
\text { Shi et al. (2013) }\end{array}$ \\
\hline 22 & Teoria das restrições & $\begin{array}{l}\text { Diminuir as restrições dos sistemas para melhorar } \\
\text { os serviços médicos no fluxo de pacientes. }\end{array}$ & Aguilar-Escobar et al. (2016) \\
\hline
\end{tabular}

Fonte: Elaboração própria

Foram várias as boas práticas analisadas, tendo destaque o uso da tecnologia da informação, citado por quatro diferentes autores, para melhorar a comunicação e os processos logísticos, além do sistema just-intime, citado por três diferentes autores, para reduzir os estoques e desperdícios.

\section{CONSIDERAÇÕES FINAIS}

O tema healthcare é discutido na literatura, porém ainda há poucos trabalhos com enfoque nas operações da logística hospitalar, apesar dos gastos neste setor representarem mais de 30\% das despesas de um hospital.

A base Scopus foi destaque na indexação dos trabalhos, representando uma contribuição de 56,8\%. Entretanto, as demais bases também apresentaram trabalhos relevantes. 
Esta pesquisa se limitou a utilização de quatro bases de dados distintas o que não abrange todo o universo de bases disponíveis. Outra limitação foi o período da amostra que contemplou os últimos doze anos. Pelo fato das limitações descritas os resultados não foram exaustivos, mas permitem a configuração de um panomara geral.

As principais abordagens sobre o tema incluiram os dois grupos no ambiente hospitalar, o assistencial com apresentação da gestão lean para fluxo de pacientes e modelos computacionais para predizer mortalidade intra-hospitalar; além do administrativo com a discussão sobre ponto de reabastecimento, separação de materiais em redes, contratação de serviços de terceiros, utilização de rôbos móveis, armários eletrônicos, RFID e tecnologia da informação para facilitar os processos logísticos e ainda técnicas e ferramentas como just-in-time, brainstormig, feedback, método Taguchi, dentre outros.

Apesar da variedade das ferramentas, técnicas e práticas utilizadas nos processos logísticos de um hospital, percebe-se, com a investigação realizada neste estudo, que os estudos de casos apresentados ocorreram basicamente em países do primeiro mundo, cenário que não corresponde à realidade mundial. Nesse sentido, pode-se situar a efetiva contribuição deste artigo mediante a identificação de uma grande lacuna no âmbito da pesquisa em logística hospitalar no Brasil e demais países periféricos.

O fato é que grande parte das pesquisas acadêmicas realizadas sobre healthcare não tem efetiva aplicação no cenário brasileiro, porque são muito diferentes as condições e contingências dos procedimentos hospitalares, mesmo em cidades como Rio de Janeiro e São Paulo, as principais do país. Nessa perspectiva, a presente pesquisa permite perceber um hiato entre a contribuição acadêmica na área e a possibilidade de implantação desses avanços em hospitais brasileiros.

Consequentemente, este artigo traz em seu bojo a necessidade e relevância de pesquisas situadas na realidade brasileira, a fim de que se amplie as possibilidades de efetiva aplicação prática dos avanços e desenvolvimentos no âmbito das inovações em healthcare.

Portanto, é proposto para trabalhos futuros a análise dos processos logísticos em países emergentes ou de terceiro mundo, como o Brasil, de forma a retratar na literatura os processos e ferramentas aplicadas para este público. 


\section{REFERÊNCIAS}

AGUIAR, F. C.; MENDES, V. L. P. S. Comunicação organizacional e Tecnologias da Informação e Comunicação (TIC) na gestão hospitalar. Perspectivas em Ciência da Informação, v. 21, n. 4, p. 138-155, 2016.

AGUILAR-ESCOBAR, V. G.; BOURQUE, S.; GODINO-GALLEGO, N. Hospital kanban system implementation: Evaluating satisfaction of nursing personnel. Investigaciones Europeas de Dirección y Economía de la Empresa, v. 21, n. 3, p. 101-110, 2015.

AGUILAR-ESCOBAR, V. G.; GARRIDO-VEGA, P. ; GONZÁLEZ-ZAMORA, M. M. Applying the theory of constraints to the logistics service of medical records of a hospital. European Research on Management and Business Economics, v. 22, n. 3, p. 139-146, 2016.

AUBRY, M.; RICHER, M-C.; TREMBLAY, M. L. Governance performance in complex environment: The case of a major transformation in a university hospital. International Journal of Project Management, v. 32, p. 1333-1345, 2014.

BAILEY, G.; CHERRETT, T.; WATERSON, B.; BREEN, L.; LONG, R. Boxed up and locked up, safe and tight! Making the case for unattended electronic locker bank logistics for an innovative solution to NHS hospital supplies (UK). International Journal of Procurement Management, v. 8, n. 1-2, p. 104-125, 2015.

BIBENG - Biblioteca da Escola de Engenharia da UFRGS - "Scopus - Tutorial". Disponível em: <http://www. ufrgs.br/bibeng/wp-content/uploads/2014/02/SCOPUS.pdf>. Acesso em: 23 jun. 2016.

BLOSS, R. Mobile hospital robots cure numerous logistic needs. Industrial Robot: An International Journal, v. 38, n. 6, p. 567-571, 2011.

BRUNERO, S.; LAMONT, S. The process, logistics and challenges of implementing clinical supervision in a generalist tertiary referral hospital. Scandinavian Journal of Caring Sciences, v. 26, n. 1, p. 186-193, 2012.

CALDERON, C. A. A.; MOHAN, E. R.; SIN NG, B. Development of a hospital mobile platform for logistics tasks. Digital Communications and Networks, p. 102-111, 2015.

CHIARINI, A. Waste savings in patient transportation inside large hospitals using lean thinking tools and logistic solutions. Leadership in Health Services, v. 26, n. 4, p. 356-367, 2013.

DESLANDES, S. F. Concepções em pesquisa social: articulações com o campo da avaliação em serviços de saúde, 1997. 
EKESKÄR, A.; RUDBERG, M. Third-party logistics in construction: the case of a large hospital Project. Construction Management and Economics, v. 34, n. 3, p. 174-191, 2016.

FERREIRA, N. S. A. As pesquisas denominadas “Estado da Arte”. Educação \& Sociedade, ano 23, n. $79,2002$.

GAO. Content analysis: a methodology for structuring and analyzing written material. United States General Accountability Office. Disponível em: <http://archive.gao.gov/f0102/157490.pdf>. Acesso em: 18 jun. $2016,1996$.

GOMES, J.; ROMÃO, M.; CARVALHO, H. Successful IS/IT projects in Healthcare: Pretesting a questionnaire.

Procedia Computer Science, v. 100, p. 375-382, 2016.

GUIMARÃES, C. M.; CARVALHO, J. C.; MAIA, A. Vendor managed inventory (VMI): evidences from lean deployment in healthcare. Strategic Outsourcing: An International Journal, v. 6, n. 1, p. 8-24, 2013.

KETELHÖHN, N.; SANZ, L. Healthcare management priorities in Latin America: Framework and responses. Journal of Business Research, v. 69, p. 3835-3838, 2016.

KRIEGEL, J.; JEHLE, F.; DIECK, M.; MALLORY, P. Advanced services in hospital logistics in the German health service sector. Logistics Research, v. 6, n. 2-3, p. 47-56, 2013.

KRIEGEL, J.; JEHLE, F.; MOSER, H.; TUTTLE-WEIDINGER, L. Patient logistics management of patient flows in hospitals: A comparison of Bavarian and Austrian hospitals. International Journal of Healthcare Management, v. 9, n. 4, p. 257-268, 2016.

KUDUVALLI, M.; GRAYSON, A. D.; AU, J.; GROTTE, G.; BRIDGEWATER, B.; FABRI, B. M. A multi-centre additive and logistic risk model for in-hospital mortality following aortic valve replacement. European Journal of Cardio-thoracic Surgery, v. 31, n. 4, p. 607-613, 2007.

KURIAN, A.; GALLAGHER, S.; CHEEYANDIRA, A.; JOSLOFF, R. Predictors of in-hospital length of stay after laparoscopic ventral hernia repair: results of multivariate logistic regression analysis. Surgical endoscopy, $v$. 24 , n. 11, p. 2789-2792, 2010.

LAPIERRE, S. D.; RUIZ, A.B. Scheduling logistic activities to improve hospital supply systems. Computers and Operations Research, v. 34, n. 3, p. 624-641, 2007.

LAW, K. M. Y. How schedule issues affect drug logistics operations: an empirical study in hospitals in China.

Industrial Management \& Data Systems, v. 116, n. 3, p. 369-387, 2016. 
LEE, S. The role of hospital selection in ambulance logistics. IIE Transactions on Healthcare Systems Engineering, v. 4, n. 2, p. 105-117, 2014.

LIAO, H-C.; CHANG, H-H. The optimal approach for parameter settings based on adjustable contracting capacity for the hospital supply chain logistics system. Expert Systems with Applications, v. 38, p. 4790-4797, 2011.

LUDWIG, A. C. W. Fundamentos e prática de metodologia científica. 2. ed. Petrópolis: Vozes, p. 124, 2012.

PAN, Z. X. T.; POKHAREL, S. Logistics in hospitals: a case study of some Singapore hospitals. Leadership in Health Services, v. 20, n. 3, p. 195-207, 2007.

PATEL, K. K.; CUMMINGS, S.; SELLIN, J.; SCOTT, L.; EL-SERAG, H. B. Applying Lean Design Principles to a Gastrointestinal Endoscopy Program for Uninsured Patients Improves Health Care Utilization. Clinical Gastroenterology and Hepatology, v. 13, n. 9, p. 1556-1559, 2015.

PEREIRA, L. C. B. Uma reforma gerencial da administração pública no Brasil. Revista do Serviço Público, v. 49, n. 1, p. 5-42, 2014.

RAIMUNDO, E. A.; DIAS, C. N.; GUERRA, M. Logística de medicamentos e materiais em um hospital público do Distrito Federal. Revista de Administração Hospitalar e Inovação em Saúde - RAHIS, v. 12, n. 2, 2015.

REIS, A. C. Product Variety Management: a Conceptual Framework and empirical studies. Tese (Doutorado) - Programa de Pós-Graduação em Engenharia de Produção, PUC-RJ, Rio de Janeiro, 2013.

REIS, A. C.; SCAVARDA, L. F.; PANCIERI, B. M. Product variety management: A synthesis of existing research. African Journal of Busines Management, v. 7, n. 1, p. 39-55, 2013.

ROWLEY, J.; SLACK, F. Conducting a literature review. Management Research News, v. 27, n. 6, p. 31-39, 2004.

SALEMA, G.; BUVIK, A. The impact of buyer-supplier integration on supplier logistics performance in the hospital sector in Tanzania: The moderation effect of buyers' cross functional integration, International Journal of Procurement Management, v. 9, n. 2, p. 166-184, 2016.

SHI, H.-Y., HWANG, S.-L., LEE, K.-T., LIN, C.-L. In-hospital mortality after traumatic brain injury surgery: A nationwide population-based comparison of mortality predictors used in artificial neural network and logistic regression models. Journal of Neurosurgery, v. 118, n. 4, p. 746-752, 2013. 
SOUZA, A. A.; PEREIRA, A. C. C.; XAVIER, A. G.; XAVIER, D. O.; MENDES, E. S. Logística hospitalar: um estudo de caso diagnóstico das dificuldades na gestão logística do setor de Engenharia Clínica. Revista Eletrônica de Administração - REA, v. 12, n. 1, 2013.

SOUZA, M. T.; SILVA, M. D.; CARVALHO, R. Revisão integrativa: o que é e como fazer. Einstein, v. 8, p. 102-106, 2010.

STENDER, G. H. C. Lean Health Care: Modelo de Implantação da Ferramenta Kanban a um Almoxarifado de um Hospital Federal no Rio De Janeiro. Dissertação (Mestrado) - Programa de Pós-Graduação em Engenharia de Produção e Sistemas, CEFET/RJ, Rio de Janeiro, 2016.

SU, S-I. I.; GAMMELGAARD, B.; YANG, S-L. Logistics innovation process revisited: insights from a hospital case study. International Journal of Physical Distribution \& Logistics Management, v. 41, n. 6, p. 577-600, 2011.

VAN DE KLUNDERT, J.; MULS, P; SCHADD, M. Optimizing sterilization logistics in hospitals. Health Care Management Science, v. 11, n. 1, p. 23-33, 2008.

VAN LENT, W. A M.; SANDERS, E. M.; VAN HARTEN, W. H. Exploring improvements in patient logistics in Dutch hospitals with a survey. BMC Health Services Research, v.12, n. 1, p. 232, 2012.

VILLA, S.; PRENESTINI, A.; GIUSEPI, I. A framework to analyze hospital-wide patient flow logistics: Evidence from an Italian comparative study. Health Policy, v. 115, p. 196-205, 2014.

VOLLAND, J.; FÜGENER, A.; SCHOENFELDER, J.; BRUNNER, J. O. Material logistics in hospitals: A literature review. Omega, v. 69, p. 82-101, 2017a.

. A column generation approach for the integrated shift and task scheduling problem of logistics assistants in hospitals. European Journal of Operational Research, v. 260, p. 316-334, 2017b.

WAHAB, A. A. O. A.; KEFELI, Z. Projecting a Long Term Expenditure Growth in Healthcare Service: A Literature Review. Procedia Economics and Finance, v. 37, p. 152-157, 2016.

ZHANG, L.; RUNZHEIMER, K.; BONIFER, E.; KEULERS, A.; PIECHOWIAK, E.; MAHNKEN, A. Improving Efficiency of Interventional Service by Lean Six Sigma. Journal of the American College of Radiology, v. 12, n. 11, p. 1200-1203, 2015. 